free survival (PFS1) from diagnosis was 19.3 months (95\% confidence interval $[\mathrm{CI}]=17.9-20.6$ months). Median OS was 50 months ( $95 \% \mathrm{CI}=42.6-57.4$ months). On univariate analysis, PFS1, primary surgery, optimal cytoreduction, complete response to primary therapy, 3 weekly chemotherapy and PFS1 interval were important predictors of OS. On multivariate analysis-response at primary treatment (CR vs. non-CR, hazard ratio $[\mathrm{HR}]=0.47[95 \% \mathrm{CI}=0.29-0.74], \mathrm{p}=0.001)$, type of relapse (clinical vs. biochemical, HR=1.74 [95\% CI=1.19-2.52], $\mathrm{p}=0.004$ ), treatment at relapse (observation vs. chemotherapy, $\mathrm{HR}=1.09[95 \% \mathrm{CI}=0.99-3.6], \mathrm{p}=0.05)$, type of chemotherapy at relapse (paclitaxel + carboplatin vs. non-paclitaxel + carboplatin, $\mathrm{HR}=0.57[95 \% \mathrm{CI}=0.36-0.91], \mathrm{p}=0.018)$ and response to relapse-1 treatment (progressors vs. non progressors, $\mathrm{HR}=2.5$ [95\% CI=1.64-3.8], $\mathrm{p}<0.001)$ were predictors of OS. Conclusion: Response at primary treatment and chemotherapy response at relapse are important predictors of OS.

Poster (014)

Epithelial Ovarian Cancer including Borderline Tumor https://doi.org/10.3802/jgo.2021.32.S1.014

\section{Prognostic value of baseline and 3 months postoperative circulating tumor DNA (ctDNA) in ovarian cancer patients}

\section{Yoo-Na Kim, ${ }^{1}$ Jinho Heo, ${ }^{2}$ Jung-Yun Lee, ${ }^{1, *}$ Seung-Tae Lee, ${ }^{2}$ Saeam Shin, ${ }^{2}$ Sunghoon Kim ${ }^{1}$}

'Department of Obstetrics and Gynecology, Yonsei University College of Medicine, Seoul, Korea (jungyunlee@yuhs.ac)

2Department of Laboratory Medicine, Yonsei University College of Medicine, Seoul, Korea

Objective: Blood sample based circulating tumor DNA (ctDNA) samples are easily obtained and can be used for disease monitoring in ovarian cancer patients undergoing primary surgery.

Methods: Patients undergoing debulking surgery for primary diagnosis or recurrent ovarian cancer were prospectively enrolled since October 2019. Control patients undergoing surgery for benign ovarian mass with CA-125 above 35 were also enrolled. Whole blood samples for cell free DNA analysis were collected immediately before and 3 months after surgery. Custom gene panel covered 9 genes (TP53, BRCA1, BRCA2, ARID1A, KRAS, $M Y C$, and $P I K 3 C A)$. Prepared libraries were sequenced using NextSeq550Dx System (Illumina) and analyzed using the custom analysis pipeline (Dxome). Clinical information was also obtained. Results: A total of 170 patients including 78 patients with epithelial ovarian cancer and 92 control patients with benign or borderline ovarian mass were analyzed. None of the control patients showed detectable ctDNA preoperatively. Analysis of baseline sample showed different ctDNA patterns with respect to histological subtypes. Based on the combination of baseline and 3 months follow up ctDNA, ovarian cancer patients were grouped to non-detected ( $\mathrm{n}=18)$, zero conversion $(\mathrm{n}=41)$, and persistently elevated group $(\mathrm{n}=19)$. Analysis of time to progression with Kaplan-Meier curve showed that the persistently elevated group patients had worse prognosis compared to other groups. Conclusion: ctDNA is a highly specific test for detecting ovarian cancer among patients with suspected ovarian mass on preoperative radiological and biomarker-based evaluation. Despite the variation in mutational landscape among histological subtypes, analysis of baseline and 3 months follow up ctDNA demonstrates a prognostic value.

Poster (015)

Gynecologic Pathology, Genetics and Epidemiology

https://doi.org/10.3802/jgo.2021.32.S1.015

\section{Putative N-glycoprotein markers of ovarian cancer from $\mathrm{N}$-glycoproteomics characterization of the whole cell lysate}

\author{
Ying Zhou, ${ }^{1}$ Zhengjian Feng, ${ }^{2}$ Nengming Lin ${ }^{1, *}$ \\ 'Hangzhou First People's Hospital, Hangzhou, China (Inm03@26.com) \\ ${ }^{2}$ Nanjing Medical University, Nanjing, China
}

Objective: To study the comparative $\mathrm{N}$-glycomics of $\mathrm{N}$-glycans on the cell-surface of ovarian cancer SKOV3 cells vs. the noncancerous ovarian epithelial IOSE80 cells.

Methods: Both SKOV3 and IOSE80 cells were cultured and extracted the proteins. With sequential digestion by trypsin, enriched by Zwitterionic Hydrophilic Interaction chromatography (ZIC-HILIC), the intact $\mathrm{N}$-glycopeptides were then isotopic diethyl labeled and analyzed using C18-RPLC-MS/MS (HCD). Comprehensive structure- and site-specific $\mathrm{N}$-glycoproteomics characterization for both the SKOV3 and IOSE80 cells were searched by GPSeeker database.

Results: Totally 13,822 intact glycopeptides from 2,918 $\mathrm{N}$-glycoproteins with comprehensive structure and site information were identified. A total of 3,733 peptide backbones and 3,754 N-glycosites were confirmed by information of GlcNAccontaining site-determining ions and structure-diagnostic. Seven hundred forty-six differentially expressed intact $\mathrm{N}$-glycopeptides in SKOV3 relative to IOSE80 cells was found, where 421 were upregulated and 325 were downregulated, respectively. Conclusion: Linking observation of differentially expressed $\mathrm{N}$-glycosylation as well as specific N-glycan substructures with epigenetic functions in ovarian cancer might help to develop the novel markers applied in diagnosis and prognosis of ovarian cancer. 